\section{Efectos de la soledad en la salud de las personas mayores}

\section{Effects of loneliness on the health of the elderly}

Elena Palma-Ayllón?

María Dolores Escarabajal-Arrieta2,*

1. Graduada en Enfermería. Sistema Andaluz de Salud. España.

2. Doctora en Psicología. Universidad de Jaén. Jaén. España.

*Autor para correspondencia.

Correo electrónico: descara@ujaen.es (María Dolores Escarabajal-Arrieta).

Recibido el 30 de julio de 2019; aceptado el 30 de septiembre de 2019.

\section{RESUMEN}

Objetivos: Identificar las consecuencias del sentimiento de soledad en la salud psicológica y física de las personas mayores. Metodología: Se consultaron las bases de datos PubMed y Web of Science. Se obtuvieron más de 2400 resultados en las búsquedas, de los cuales se seleccionaron

10 artículos que cumplían los criterios de selección establecidos.

Resultados: Se identifican como consecuencias del sentimiento de soledad en la salud de las personas mayores la presencia de trastornos psicológicos (ansiedad y depresión); también es un factor predictor de deterioro cognitivo y produce un mayor riesgo de mortalidad, entre otros. Conclusiones: Dados los efectos tan negativos de la soledad en

las personas mayores, es necesario realizar un abordaje preventivo y multidisciplinar para minimizar las alteraciones en la salud.

PALABRAS CLAVE: Adultos mayores, soledad, salud.

\section{ABSTRACT}

Objectives: Identify the consequences of the feeling of loneliness in the psychological and physical health of the elderly. Methodology: The PubMed and Web of Science databases were consulted. We obtained more than 2400 results in the searches from which 10 articles were selected that met the established selection criteria. Results: Psychological disorders (as anxiety and depression) are identified as consequence of the feeling of loneliness in the health of the elderly, is too a predictive factor of cognitive impairment and producing a greater risk of mortality, among other. Conclusions: Given the negative effects of loneliness in the elderly, it is necessary to carry out a preventive and multidisciplinary approach to minimize alterations in health.

KEYWORDS: Older adults, loneliness, health.

\section{- INTRODUCCIÓN}

En los países desarrollados, el descenso de la natalidad y el aumento de la esperanza de vida causados por los progresos sociosanitarios han hecho del envejecimiento poblacional una realidad dominante. Se estima que, en unos 40 años, la población de personas mayores de 60 años se duplicará en el mundo. Este envejecimiento de la población se ha convertido en un serio desafío económico, social y sanitario ${ }^{1}$. Además, se ha observado que este envejecimiento trae consigo condiciones bajo las cuales las personas se sienten más solas ${ }^{1}$. El sentimiento de soledad, aunque puede afectar a las personas en cualquier etapa del desarrollo vital, tiene una incidencia especialmente elevada entre las de menos de 25 años y las mayores de $65^{2}$.

Las personas de edad avanzada se encuentran particularmente expuestas a eventos que suelen acarrear una enorme transformación en sus relaciones sociales, haciéndolas más vulnerables a la soledad. En este sentido, podemos encontrar diversos factores asociados con este sentimiento: el género, la edad, la situación laboral, el nivel de estudios, los recursos económicos, el estado de salud y residir en el medio rural o en el urbano ${ }^{3}$. Entre todos ellos destacan dos factores de riesgo fundamentales: la defunción de la pareja y el deterioro de la salud ${ }^{4}$.

Las discapacidades físicas y las enfermedades mentales son desencadenantes del sentimiento de soledad entre las personas mayores ${ }^{5}$, sobre todo si viven solas ${ }^{6}$, lo que implicaría que salud y soledad están asociadas de forma negativa. Además, algunas investigaciones han ahondado en estos componentes, indicando que esta relación se produce en sentido inverso, siendo la soledad un factor de riesgo para que la salud empeore?
Es aquí donde surge la necesidad de llevar a cabo esta revisión, ya que, si bien existe evidencia sobre la influencia negativa de la soledad en la salud y viceversa, hay pocos estudios de revisión cuya población de estudio sean personas mayores, en los que, además, se profundice acerca de qué consecuencias conlleva el sentimiento de soledad en la salud tanto a nivel físico como mental en este colectivo.

El sentimiento de soledad, siguiendo a Perlman y Peplau (1981), es una experiencia desagradable que ocurre cuando la red de relaciones sociales de la persona es deficiente en algún aspecto importante, cuantitativa o cualitativamente ${ }^{8}$. Según De Jong Gierveld (1987), las personas sufren la soledad al percibir déficit en determinadas relaciones; por ejemplo, cuando su número resulta más escaso del que consideran deseable o cuando no aportan la intimidad que necesitan'. Ambas definiciones, aunque clásicas, continúan siendo aceptadas y son empleadas con frecuencia en la literatura internacional.

Es importante diferenciar el sentimiento de soledad de otros términos relacionados que, en ocasiones, pueden haber sido considerados sinónimos, ya que, por ejemplo, vivir solo no es necesariamente indicativo de soledad, al mantenerse igualmente contacto social frecuente y una participación social activa en las organizaciones comunitarias.

Por otro lado, es importante también diferenciar el sentimiento de soledad de la experiencia de "estar a solas". Esta última refleja un estado de aislamiento social que implica un distanciamiento voluntario de la propia red social, mientras que la soledad es involuntaria y se asocia estrechamente con los déficits en la calidad percibida de las interacciones sociales que se poseen. También es necesario diferenciar las ideas relacionadas con el aislamiento social del sentimiento de soledad en sí 
mismo. El aislamiento social se define como el estado objetivo en el que el contacto social con otras personas es mínimo, mientras que la soledad reflejaría un estado subjetivo de carencia de afecto y cercanía deseados ${ }^{10}$.

En cuanto a su prevalencia, la soledad entre las personas mayores varía según los estudios en función de la herramienta de medida utilizada, las poblaciones estudiadas, el grupo de edad y los tamaños de muestra considerados. Así, Arsenijevic y Groot ${ }^{11}$ recopilaron entre 2004 y 2013 datos acerca de los niveles de soledad entre los mayores en 10 países europeos. En el ańo 2013, la menor tasa de prevalencia la encontramos en Dinamarca $(10 \%)$ y la mayor en Italia $(33,4 \%)$, seguida por Francia $(30,2 \%)$, Bélgica $(25,0 \%)$ y Suecia $(22,1 \%)^{11}$. Otro estudio, realizado por Nicolaisen y Thorsen², estimó que el 30,2\% de los adultos noruegos mayores de 65 años sufren la soledad ${ }^{2}$.

En España, la prevalencia de soledad es menor que en estos países; se ha estimado que un $21,4 \%$ de los adultos mayores españoles la padecen ${ }^{11}$.

\section{Objetivos}

El objetivo general de este trabajo es realizar una revisión para identificar las consecuencias del sentimiento de soledad en la salud psicológica y física de las personas mayores.

\section{Métodos}

Esta revisión sistemática se inició realizando una búsqueda bibliográfica en las bases de datos biomédicas PubMed y Web of Science. Se utilizó el operador booleano AND en cada búsqueda, junto con las palabras clave: "adultos mayores", "soledad" y "salud", y en el caso de las búsquedas en términos ingleses: "older adults", "loneliness" y "health".

Para la selección de los artículos se determinaron los siguientes criterios de inclusión: artículos publicados entre 2014 y 2019, escritos en idioma inglés o español y disponibles con el texto íntegro.

Por otro lado, los criterios de exclusión establecidos en esta revisión fueron los siguientes: artículos cuya población de estudio fuera menor de 60 años, artículos de revisión, artículos que no tratasen las consecuencias del sentimiento de soledad, sino de términos similares (aislamiento social, etc.), artículos duplicados y todos aquellos que no se ajustasen a los objetivos planteados en la revisión.

Se explican a continuación, de forma detallada, los resultados obtenidos en cada base de datos y el proceso de selección de artículos.

\section{PubMed}

Establecidos los criterios de inclusión, en la búsqueda con las palabras clave en español se obtiene tan solo un artículo. Este no se selecciona por no ajustarse a ningún objetivo de nuestro trabajo.

En cuanto a la búsqueda con las palabras clave en inglés, se obtienen 170 artículos, de los cuales se seleccionan 15 por ser potencialmente relevantes por título y resumen. Tras una lectura completa de estos se seleccionan 3 artículos.

\section{Web of Science}

Establecidos los criterios de inclusión, en la búsqueda con las palabras clave en español se obtiene 1 artículo, que no es seleccionado por no ajustarse a los objetivos de la revisión.

En cuanto a la búsqueda con las palabras clave, en inglés se obtienen 399 artículos, de los cuales se seleccionan 17 por ser potencialmente relevantes por título y resumen. Tras una lectura completa de estos se seleccionan 7 artículos.
Número de artículos identificados

mediante las búsquedas en bases de datos

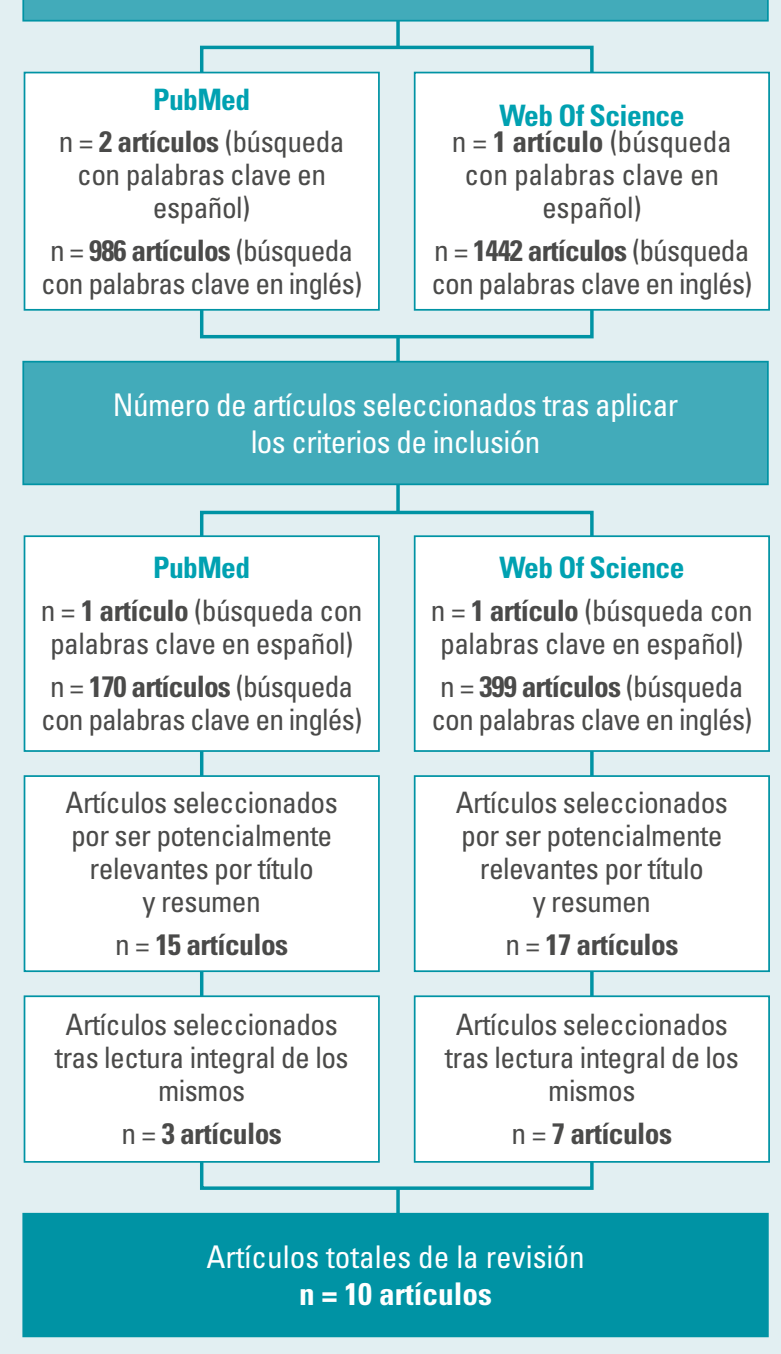

Figura 1. Procedimiento de selección de artículos.

En la figura 1 se describe el proceso de selección de los artículos expuesto con anterioridad, donde se parte del global de 2431 artículos hasta llegar a los 10 artículos que forman parte de los analizados en este trabajo.

\section{RESULTADOS}

Tras la revisión y lectura integral de los artículos se obtienen los siguientes resultados:

- El artículo de Dahlberg et al. ${ }^{12}$ asocia el sentimiento de soledad a trastornos psicológicos como la ansiedad, el nerviosismo o la angustia.

- Los artículos de Gale et al. ${ }^{13}$ y Gerino et al. ${ }^{14}$ asocian la soledad con un mayor riesgo de padecer depresión. El primero de ellos ańade, además, un mayor riesgo de fragilidad física, mientras que el segundo 
suma, además, la asociación con la ansiedad y una menor calidad física.

- Los estudios de Zhong et al. ${ }^{15,16}$ y los de Donovan et al. ${ }^{17,18}$ coinciden en sus resultados al identificar la soledad como un factor predictor de deterioro cognitivo. El estudio de Donovan identifica la soledad como un síntoma neuropsiquiátrico relevante en la preclínica de la enfermedad de Alzheimer.

- Los resultados del estudio de Tormstad et al. ${ }^{19}$ muestran como consecuencias de la soledad una mayor probabilidad de padecer mala salud, sentimientos de desesperanza, sufrir una enfermedad crónica, recibir ayuda para las actividades de la vida diaria y presentar insatisfacción con la vida.

- Finalmente, los trabajos de Chan et al. ${ }^{20}$ y Luo et al. ${ }^{21}$ asocian el sentimiento de soledad con un mayor riesgo de mortalidad.

\section{DISCUSIÓN}

El sentimiento de soledad es una experiencia negativa que se percibe como una carencia emocional, ya sea porque las relaciones sociales que mantiene la persona son menores de las que serían deseables o porque no percibe el apoyo emocional suficiente de estas. Lamentablemente, este sentimiento tiene una elevada prevalencia entre la población anciana, lo que implica, a su vez, múltiples consecuencias negativas en la salud de las personas mayores. En relación con esto se ha propuesto una asociación entre este sentimiento doloroso y la aparición de trastornos psicológicos, así como su identificación como factor predictor de deterioro cognitivo.

En este sentido, los trabajos de Dahlberg et al. ${ }^{12}$, Gale et al. ${ }^{13}$, Gerino et al. ${ }^{14}$ y Tomstad et al. ${ }^{19}$ coinciden en que el sentimiento de soledad influye negativamente en la salud mental, provocando ansiedad y depresión en las personas ancianas. Por lo tanto, la soledad sería un factor de riesgo para la salud mental, especialmente, en relación con la depresión $n^{22-24}$.

Por otro lado, otros estudios identifican la depresión como un factor de riesgo para la soledad. Así, investigaciones como las de Prieto-Flores et al. ${ }^{25}$ y Fernandes et al. ${ }^{26}$ planteaban que la soledad y la depresión tienen una relación de retroalimentación, generando de esta forma un efecto sinérgico entre ambas que resulta muy difícil de combatir y que ocasiona un deterioro considerable en la persona mayor ${ }^{4}$.

Además, en los últimos ańos, el suicidio es motivo de gran preocupación, en particular entre las personas mayores de 75 años $^{27}$. Así, la depresión, junto con otros trastornos psiquiátricos son, claramente, los factores de riesgo más importantes para que se produzca el suicidio entre las personas mayores. En este sentido, la mayoría de los adultos mayores que se suicidan viven solos. Sin embargo, la relación entre la soledad y el suicidio es compleja. De hecho, el aislamiento social tiene un impacto más significativo que la soledad como tal; y el riesgo de suicidio es particularmente alto durante el año siguiente al fallecimiento del cónyuge, siendo la pérdida del ser querido uno de los eventos de la vida más estresantes para la persona anciana ${ }^{27}$.

Por otro lado, en cuanto a la soledad como factor predictor de deterioro cognitivo, los trabajos de Zhong et al. ${ }^{15,16} \mathrm{y}$ los de Donovan et al. ${ }^{17,18}$ coinciden en esta propuesta. En concreto, el estudio de Donovan et al. ${ }^{18}$ identifica la soledad como un síntoma neuropsiquiátrico relevante en la preclínica de la enfermedad de Alzheimer. En relación con esto, estudios previos ${ }^{28,29}$ respaldan la relación entre soledad, deterioro cognitivo y enfermedad de Alzheimer. Además, las personas con demencia de Alzheimer se caracterizan por ser más dependientes y vulnerables, tanto en los aspectos sociales como en los relacionados con la salud física y mental, lo que supone una serie de importantes desafíos para la sociedad y el sistema sanitario ${ }^{30}$.

Por otra parte, tal y como se ha indicado para la relación entre soledad y depresión, también se ha informado de una relación bidireccional entre la soledad y el deterioro cognitivo ${ }^{15}$. Este hecho podría implicar que los factores de soledad, demencia y depresión están interrelacionados entre sí, puesto que diversos trabajos respaldan también la relación entre depresión y demencia ${ }^{31,32}$.

En cuanto al resto de las consecuencias identificadas en los trabajos analizados, son una mayor probabilidad de padecer mala salud y la presencia de sentimientos de desesperanza, tener una enfermedad crónica, recibir ayuda para las actividades de la vida diaria, presentar una insatisfacción con la vida (Tomstad et al., ${ }^{19}$ ) y un mayor riesgo de mortalidad (Chan et al. ${ }^{20} \mathrm{y}$ Luo et al. ${ }^{21}$.

En cuanto a las características de las poblaciones de estudio, en general, los trabajos se han desarrollado con muestras grandes, pero difícilmente comparables, ya que los rangos en el número de participantes oscilaban entre 14199 y 79 personas evaluadas.

Respecto a las características sociodemográficas, cabría señalar que en todos los estudios analizados el porcentaje de mujeres fue mayor que el de hombres. También destacan los porcentajes de personas casadas, y también viudas. Además, los trabajos parecen coincidir en un elevado porcentaje de participantes con un nivel de estudios bajo. En cuanto a los porcentajes de "vivir solo/a", estos son muy variados.

Consultando la bibliografía existente, estas características coinciden con factores predictores de soledad ya identificados, como ser mujer, no estar casado/a, vivir solo/a y poseer un nivel de estudios bajo ${ }^{3,32}$. Otros factores de riesgo identificados son tener bajos ingresos económicos, relaciones sociales de baja calidad, falta de aficiones y padecer mala salud $33,32-34$

En la actualidad, la soledad se ha convertido en un problema de salud pública, no solo por ser fuente de sufrimiento y por su influencia en la salud de las personas mayores, sino también por sus importantes repercusiones en los sistemas sanitarios. En este sentido, la soledad ha sido identificada como factor social predictor de un mayor número de visitas al médico, rehospitalizaciones y un aumento de la estancia hospitalaria $^{35-37}$. Este hecho puede ser debido no solo a la relación soledad-peor salud, sino también a que las personas mayores, que se sienten solas, utilizan las visitas médicas no siempre por razones médicas, sino para satisfacer parte de sus necesidades de interacción interpersonal.

Por ello, y dadas las consecuencias negativas que provoca la soledad en la salud de las personas mayores, se hace necesaria la identificación y puesta en marcha de intervenciones orientadas a paliar la soledad entre este colectivo.

Por otra parte, aquellos adultos mayores que tienen sus necesidades de atención domiciliaria satisfechas presentan niveles más altos de satisfacción en la vida y menores niveles de soledad y estrés percibido ${ }^{38}$; por tanto, los equipos de atención primaria desempeñarían en este aspecto un papel fundamental.

El equipo de atención primaria debería promover la autonomía de estos pacientes impulsando intervenciones con el objetivo de reestructurar pensamientos sociales desadaptativos, a fin de conseguir su participación en actividades desarrolladas dentro de la comunidad que favorezcan su socialización. En este sentido, cabe señalar que las actividades más efectivas para actuar sobre la soledad son las actividades grupales, así como otras con un enfoque participativo que incluyan también a personas que no presenten este mismo problema.

Es importante, además, que reciban apoyo emocional. Estas actividades tienen un mayor impacto si se realizan en colaboración con el resto 
de los servicios sociales y sanitarios del territorio, y con las asociaciones civiles y religiosas de la comunidad ${ }^{39}$.

Todo ello hace necesario seguir investigando para ampliar y profundizar en la efectividad de las intervenciones y sus beneficios, de manera preventiva, en la salud de las personas mayores.

\section{- CONCLUSIONES}

Las condiciones sociales han llevado a que cada vez más personas estén solas de forma involuntaria, acrecentando el sentimiento de soledad como una experiencia negativa. Este aspecto cobra especial importancia entre las personas mayores, ya que se constatan múltiples consecuencias graves en su salud.

Algunas de estas alteraciones vinculadas con la soledad son su asociación con trastornos psicológicos, como la ansiedad y la depresión, y su relación predictiva con la presencia de deterioro cognitivo.

La soledad es un problema de salud pública, genera sufrimiento y enfermedad en las personas mayores, a lo que se suma su efecto en los sistemas sanitarios. Por todo ello, es importante desarrollar e implementar intervenciones con el objetivo de paliarla y prevenir sus efectos negativos

\section{Conflicto de intereses}

Las autoras declaran no tener ningún conflicto de intereses.

\section{BIBLIOGRAFÍA}

1. Bandari R, Klankeh HR, Shahboulaghi FM, Ebadi A, Keshtkar AA, Montazeri A. Defining loneliness in older adults: protocol for a systematic review. Syst Rev. 2019;8(1):26

2. Nicolaisen $M$, Thorsen K. Who are lonely? Loneliness in different age groups (18-81 years old), using two measures of loneliness. Int J Aging Hum Dev. 2014;78:229-57.

3. Cohen-Mansfield J, Hazan H, Lerman Y, Shalom V. Correlates and predictors of loneliness in older-adults: a review of quantitative results informed by qualitative insights. Int Psychogeriatr. 2016 28:557-76

4. Doblas JL, Díaz- Conde MP. Viudedad, soledad y salud en la vejez. Rev Esp Geriatr Gerontol. 2018:53(3):128-33.

5. Burholt V, Scharf T. Poor health and loneliness in later life: The role of depressive symptoms, social resources, and rural environments. J Gerontol B Psychol Sci Soc Sciences. 2014:69:311-24.

6. Sundström G, Fransson E, Malmberg B, Davey A. Loneliness among older Euro-peans. Eur J Ageing. 2009;6:267-75

7. Losada A, Márquez-González M, García-Ortíz L, Gómez-Marcos MA, Fernández-Fernández V Rodríguez-Sánchez E Loneliness and mental health in a representative sample of community-dwelling Spanish older adults. J Psychol. 2012;146:277-92.

8. Perlman D. Peplau LA. Toward a social psychology of loneliness. En: Duck S, Gilmour R, eds. Personal relationships in disorder. London: Academic Press; 1981. p. 31-56.

9. De Jong Gierveld J. Developing and testing a model of loneliness J Pers Soc Psychol. 1987:53:119-28.

10. Ong AD, Uchino BN, Wethington E. Loneliness and health in olde adults: a mini-review and synthesis. Gerontology. 2016;62(4):443-9.

11. Arsenijevic J, Groot W. Does household help prevent loneliness among the elderly? An evaluation of a policy reform in the Netherlands. BMC Public Health. 2018;18:1104.

12. Dahlberg L, Agahi N, Lennartsson C. Lonelier tan ever? Loneliness of older people over two decades. Arch Gerontol Geriatr. 2018:75:96-103

13. Gale CR, Westbury L, Cooper C. Social isolation and loneliness as risk factors for the progression of frailty: the English Longitudina Study of Ageing. Age Ageing. 2018;47(3):392-7.

14. Gerino E, Rollè L, Sechi C, Brustia P. Loneliness, resilience, mental health, and quality of life in old age: a structural equation mode Front Psychol. 2017:8:2003.
15. Zhong BL, Chen SL, Tu X, Conwell Y. Loneliness and cognitive function in older adults: findings from the Chinese longitudinal healthy longevity survey. J Gerontol B Psychol Schi Soc Sci. 2017;72(1):120-8

16. Zhong BL, Chen SL, Conwell Y. Effects of transient versus chronic Ioneliness on cognitive function in older adults: findings from the Chinese longitudinal healthy longevity survey. Am J Geriatr Psychiatry. 2016;24(5):389-98.

17. Donovan NJ, Wu Q, Rentz DM, Sperling RA, Marshall GA, Glymou MM. Loneliness, depression and cognitive function in older U.S adults. Int J Geriatr Psychiatry. 2017:32(5):564-73.

18. Donovan NJ, Okereke OI, Vannini P, Amariglio RE, Rentz DM, Marshall GA, et al. Association of higher cortical amyloid burden with Ioneliness in cognitively normal older adults. JAMA Psychiatry. 2016;73(12):1230-7

19. Tomstad S, Dale B, Sundsli K, Saevareid HI, Söderhamm U. Who often feels lonely? A cross- sectional study about loneliness and its related factors among older home-dwelling people. Int J Olde People Nurs. 2017:12(4):1-10.

20. Chan A, Prassana R, Ma S, Malhotra R. Loneliness and all-caus mortality in community- dwelling elderly Singaporeans. Demographic Research. 2015;32:1361-82.

21. Luo Y, Waite LJ. Loneliness and mortality among older adults in China. J Gerontol B Psychol Sci Soc Sci. 2014;69(4):6333-45

22. Courtin E, Knapp M. Social isolation, loneliness and health in old age: a scoping review, Health Soc Care Community. 2017; 25(3):799-812.

23. Cacioppo JT, Hawkley LC, Thisted RA. Perceived social isolation makes mesad: 5 -year cross-lagged analyses of Ioneliness and depressive symptomato-logy in the Chicago Health, Aging, and Social Relations Study. Psychol Aging. 2010;25:453-63

24. Wang J, Mann F, Lloyd-Evans B, Ma R, Johnson S. Association between loneliness and perceived social support and outcomes of mental health problems: a systematic review. BMC Psychiatry. 2018:8:156

25. Prieto-Flores M, Forjaz MJ, Fernández-Mayoralas G, Rojo-Pérez F Martínez-Martin P. Factors associated with loneliness of noninstitutionalized and institutionalized older adults. J Aging Health. 2011;23(1):177-94
26. Fernandes S, Davidson JGS, Guthrie DM. Changes in social engagement and depression predict incident loneliness among seriously ill home care clients. Palliat Support Care. 2018;116(2):170-9.

27. Pitchot W. Suicide in the elderly. Rev Med Liege. 2014;69(5-6):390-4

28. Wilson RS, Krueger KR, Arnold SE, Schneider JA, Kelly JF, Barnes $\mathrm{LL}$, et al. Loneliness and risk of Alzheimer disease. Arch Gen Psychiatry. 2007;64(2):234-40.

29. Shankar A, Hamer M, McMunn A, Steptoe A Social isolation and Ioneliness: relationships with cognitive function during 4 years of follow-up in the English Longitudinal Study of Ageing. Psychosom Med. 2013;75(2):161-70.

30. Tzang RF Yang AC, Yeh HL, Liu M-E, Tasai S-J. Association of depression and loneliness with specific cognitive performance in non-demented elderly males. Med Sci Monit. 2015;21:100-4.

31. Bennett S, Thomas AJ. Depression and dementia: cause, consequence or coincidence? Maturitas. 2014:79(2):184-90.

32. Ong AD, Uchino BN, Wethington E. Loneliness and health in olde adults: a mini-review and synthesis. Gerontology. 2016;62(4):443-

33. Emerson KG, Jayawardhana J. Risk factors for loneliness in elderly adults. J Am Geriatr Soc. 2016; 64(4):886-87.

34. Arslantas H, Adana F, Abacigil Ergin F, Kayar D, Acar G. Loneliness in elderly people, associated factors and its correlation with quality of life: a field study from western Turkey. Iran J Public Health. 2015;44(1):43-50

35. Zhang J, Xu L, Li J, Sun S, Ding G, Qin W, et al. Loneliness and health service utilization among the rural elderly in Shandong, China: a cross-sectional study. Int J Environ Public Health. 2018;15(7).

36. Newall N, Mc Arthur J, Menec VH. A longitudinal examination of social participation, loneliness, and use of physician and hospital services. J Aging Health. 2015;27(3):500- 18.

37. Gerst-Emerson $\mathrm{K}$, Jayawardhana J. Loneliness as a public health issue: the impact of loneliness on health care utilization among older adults. Am J Public Health. 2015;105(5):1013-9.

38. Kadowaki L, Wister AV, Chappell NL. Influence of home care on life satisfaction, loneliness, and perceived life stress. Can J Aging. 2015;34(1):75-89.

39. Gené-Badia J, Ruiz-Sánchez M, Obiols-Masó N, Lagarda Jiménez E. Social isolation and loneliness: What can we do as Primary Care teams? Aten Primaria. 2016:48(9):604-9. 